\title{
Prediction of preterm birth with and without preeclampsia using mid-pregnancy immune and growth-related molecular factors and maternal characteristics
}

\author{
Laura L. Jelliffe-Pawlowski ${ }^{1,7} \cdot$ Larry Rand $^{2,7} \cdot$ Bruce Bedell $^{3} \cdot$ Rebecca J. Baer $\mathbb{D}^{4,7} \cdot$ Scott P. Oltman ${ }^{4,7}$. \\ Mary E. Norton ${ }^{2} \cdot$ Gary M. Shaw ${ }^{5} \cdot$ David K. Stevenson $^{5} \cdot$ Jeffrey C. Murray $^{3} \cdot$ Kelli K. Ryckman ${ }^{6}$
}

Received: 7 December 2017 / Revised: 10 February 2018 / Accepted: 7 March 2018

(c) The Author(s) 2018. This article is published with open access

\begin{abstract}
Objective: To evaluate if mid-pregnancy immune and growth-related molecular factors predict preterm birth (PTB) with and without $( \pm)$ preeclampsia.

Study design: Included were 400 women with singleton deliveries in California in 2009-2010 (200 PTB and 200 term) divided into training and testing samples at a 2:1 ratio. Sixty-three markers were tested in 15-20 serum samples using multiplex technology. Linear discriminate analysis was used to create a discriminate function. Model performance was assessed using area under the receiver operating characteristic curve (AUC).

Results: Twenty-five serum biomarkers along with maternal age $<34$ years and poverty status identified $>80 \%$ of women with $\mathrm{PTB} \pm$ preeclampsia with best performance in women with preterm preeclampsia $(\mathrm{AUC}=0.889,95 \%$ confidence interval (0.822-0.959) training; 0.883 (0.804-0.963) testing).

Conclusion: Together with maternal age and poverty status, mid-pregnancy immune and growth factors reliably identified most women who went on to have a PTB \pm preeclampsia.
\end{abstract}

Electronic supplementary material The online version of this article (https://doi.org/10.1038/s41372-018-0112-0) contains supplementary material, which is available to authorized users.

Laura L. Jelliffe-Pawlowski

Laura.Jelliffe@ucsf.edu

1 Department of Epidemiology and Biostatistics, University of California San Francisco School of Medicine, San Francisco, CA 94107, USA

2 Department of Obstetrics, Gynecology and Reproductive Sciences, University of California San Francisco School of Medicine, San Francisco, CA 94107, USA

3 Department of Pediatrics, University of Iowa School of Medicine, Iowa City, IA 52242, USA

4 Department of Pediatrics, University of California San Diego, La Jolla, CA 92093, USA

5 Department of Pediatrics, Stanford University School of Medicine, Stanford, CA 94305, USA

6 Department of Epidemiology, University of Iowa, College of Public Health, Iowa City, IA 52242, USA

7 California Preterm Birth Initiative, University of California San Francisco School of Medicine, San Francisco, California 94107, USA

\section{Introduction}

Worldwide, more than 15 million babies are born preterm (before 37 completed weeks of gestation) each year [1]. Preterm birth (PTB) and its related complications are the leading cause of death in children less than five years of age and contribute to more than one million deaths per year [2]. Survivors of PTB are more likely to suffer from both shortand long-term morbidities including blindness, deafness, neurodevelopmental delay, psychiatric disturbance, diabetes, and heart disease in later life [3-6]. While all neonates born preterm are at risk for short and long-term morbidity and mortality, those with early PTB (gestational age (GA), <32 weeks) are at the highest risk [3-8].

Despite increased clinical, research, and policy focus, rates of PTB are increasing worldwide-including in the United States [1]. After several years of decline, in 2015, the rate of PTB in the United States increased [9]. This pattern of increase continued into 2016 [10].

The continuing burden of PTB despite increased focus suggests the need for a different approach to addressing PTB from a research, clinical, and policy perspective. While historically, prevention efforts have focused mostly on 
women with a previous PTB or short cervix, or have focused on extending gestational duration in women with early signs of labor [11-13], there is a growing push for management based on a woman's specific personal risk profile. In 2016, the Society for Maternal Fetal Medicine (SMFM) released its first PTB Toolkit [14] which outlines recommended management of women based on a number of risk factors for PTB (e.g., bacteriuria, smoking, obesity, pregestational diabetes, and chronic hypertension).

Consideration of a clinical shift to address the risk of PTB has also recently begun to be considered for women testing as "high-risk" based on mid-pregnancy biomarkers [15-17]. In general, the principle behind such tests is that they might allow for the identification of at-risk pregnant women that may otherwise go unidentified. While the question of whether women with molecular risk without other traditional risks (e.g., previous PTB, short cervix) might benefit from existing therapies (e.g., progesterone, cervical pessary, cervical cerclage, tocolytic administration, and antibiotic therapy) is still unknown, all of these interventions require timely administration [18]. These efforts are closely aligned with those focused on early identification of pregnancies at increased risk for preeclampsia (ending in preterm and term birth) given the established efficacy of aspirin administration $\leq 16$-weeks for reducing recurrence [19, 20].

Recent years have seen progress in the development of PTB prediction tests with three tests in or moving into the market. Two existing tests measure proteins and microparticles identified by multiple reaction monitoring mass spectrometry $[15$, 16] and one uses circulating cell-free plasma RNAs tested by Q-PCR [17] to identify women at increased risk for spontaneous PTB. Currently these tests focus mostly on spontaneous PTB (PTB related to preterm premature rupture of membranes (PPROM) or premature labor) and generally do not address provider initiated PTB (PTB resulting from cesarean section or induction due to fetal or maternal indication). Efforts focused on molecular and other prediction testing for preeeclampsia are also well underway but also rarely address overlap with efforts aimed at predicting PTB [21, 22].

While existing prediction tests for spontaneous PTB (and for preeclampsia without a focus on PTB) demonstrate the promise of using mid-pregnancy biomarkers for prediction purposes, these tests lack a generalizability to all PTBs. Given the breadth of data demonstrating common pathophysiological underpinning across spontaneous and provider initiated subtypes of PTB including among those \pm preeclampsia [23-28], it appears possible that a predictive test could be developed that covers a wider range of PTB phenotoypes. For example, all PTB subtypes \pm preeclampsia have been shown to have strong links to markers of immune function (e.g., cytokines and chemokines) [23-26] and to angiogenic growth factors (e.g., vascular endothelial growth factor (VEGF)) [27, 28].
For this study, we hypothesized that a comprehensive test for PTB across subtypes including \pm preeclampsia could be developed using mid-pregnancy growth and immune-related factors along with maternal demographics and obstetric factors. Markers were tested in 15-20 week serum samples collected as part of routine prenatal screening with predictive performance assessed in training and testing subsets.

\section{Materials and methods}

All women included in the study are part of a populationbased cohort of all singleton California births from July 2009 through December $2010(n=757,853)$. All women had gestational dating by first trimester ultrasound and had a second trimester serum marker test done as part of routine prenatal screening for aneuploidies and neural tube defects by the California Genetic Disease Screening Program $(n=$ 241,000). Candidate cases and controls all had a second trimester serum sample banked by the California Biobank Program $(n=77,604)$ [29] and had detailed demographic and obstetric information available in a linked hospital discharge birth cohort database maintained by the California Office of Statewide Health Planning and Development (OSHPD) $(n=61,339)$. A number of previous papers have been published that leverage data and screening results for women in this and other California cohorts [30, 31]. The final source set for this study included 4025 singletons with births before 37 weeks, and 56,081 with births on or after 37 completed weeks through 44 weeks. From this set, we selected 100 PTB cases with gestational ages at birth $<32$ weeks, 100 PTB cases with gestational ages at birth from 32 to 36 weeks, and 200 term controls with gestational ages at birth from 39 to 42 weeks using simple random sampling wherein each within group pregnancy had an equal probability of selection. The resulting sample (by $<32,32-26$, and 39 to 42 weeks) were then divided into training and testing subsets at a ratio of 2:1 (Supplemental Fig. 1). This was a convenient random sample wherein total number was determined based on the financial resources available for testing.

\section{Maternal demographic and obstetric characteristics}

Demographic and obstetric factors evaluated included race/ ethnicity, maternal age, years of formal education, place of maternal birth, low-income status (as indicated by "MediCal" payment for delivery (the California health program for low-income persons (generally defined as income $<138 \%$ of the United States poverty level)), parity, preexisting diabetes, preexisting hypertension, reported smoking, obesity (body mass index (BMI) $\geq 30 \mathrm{~m} / \mathrm{kg}^{2}$ ), interpregnancy interval (IPI) $<12$ months, and previous 
PTB. All variables were derived from the OSHPD birth cohort file, which combines birth certificate records and all hospital discharge records for the mother and baby from 1 year prior to the birth to 1 year after the birth. Coding of preexisting and gestational diabetes and hypertension was based on International Classification of Diseases, 9th Revision, Clinical Modification (ICD-9-CM) [32] four digit codes contained in the cohort file.

\section{Serum biomarker testing}

Immune and growth-factor molecular testing was done using residual serum samples from second trimester (15-20 week) prenatal screening. Specimens were stored in 1 milliliter tubes at $-80^{\circ} \mathrm{C}$. Markers tested included twenty interleukins, three interferons, eleven chemokine ligands, eight members of the tumor necrosis factor-alpha (TNFA) super family cytokines, 12 growth factors, three colonystimulating factors, two soluble adhesion molecules, and leptin, plasminogen activator inhibitor-1 (PAI-1), resistin, and receptor for advanced glycosylation end products (RAGE) (see Fig. 1 for complete listing). While many of these markers have been shown to have close links to PTB or preeclampsia [24, 33-36], for this study we elected to run the full panel of immune and growth-factor related markers available via multiplex testing through our partner laboratory (the Human Immune Monitoring Center (HIMC) at Stanford University) [37] given the established interconnectedness of all of these markers to immune function and as such, the potential for revealing novel patterns and relationships-particularly given the role of immune function in pregnancy [38].

All markers were read using a Luminex 200 instrument (Austin, TX) in accordance with the manufacturer recommendations. Details regarding Luminex lab protocols at the HIMC are available on their website [37]. All markers were tested using a human multiplex kit that was purchased from Affymetrix Inc. (Santa Clara, CA) with the exception of human soluble receptors, which were measured using a Millipore high sensitivity multiplex kit (HSCRMAG32KPX14) (Billerica, MA). Median fluorescence intensity (MFI) values were reported for all markers using Masterplex software (Hitashi Solutions, San Bruno, CA). To avoid error inherent in $\log$ transformation of MFI to $\mathrm{pg} / \mathrm{mL}$, analyses relied on the MFI average, which was based on measurement of two aliquots tested on the same plate for each case and control. All inter-assay coefficients (CVs) were $<15 \%$ across all markers and all intra-assay $\mathrm{CVs}$ were $<10 \%$.

\section{Data analyses}

Simple logistic regression (including odds ratios (ORs) and their 95\% (CIs)) were used for association testing in the training set using demographic, clinical, and molecular factors (standardized using natural log transformation) and to build multivariate models. So as not to lose information that might be critical to prediction, for variable selection into multivariate models we utilized backward stepwise regression wherein all possible predictors were entered into the model and the criteria for remaining in the model was $p<0.20$. Predictors with a $p \geq 0.05$ and $<0.20$ were removed in any instance where their exclusion resulted in a $<1 \%$ decrease in the concordance statistic (cstatistic) (equivalent to the area under the receiver operating characteristic curve (AUC)). Similarly, in any instance where the variable inflation factor (VIF) indicated major multicollinearity among predictors (defined as VIF $\geq 2.5$ ) predictors were removed when their exclusion resulted in a $<1 \%$ decrease in the c-statistic. All variables in the final multivariate logistic model were included in the final linear discriminate analysis (LDA) algorithm with assessment of performance using AUC in both the training and testing subsets. AUC performance was evaluated for all PTBs and for early PTB ( $<32$ weeks) and late PTB (33-36) subgroups including in spontaneous and provider initiated subgroups and by preeclampsia diagnosis by ICD-9-CM code [32]. "Spontaneous PTBs" were considered to be those where the birth certificate or hospital discharge record noted "preterm premature rupture of membranes" (PPROM) or "preterm labor." Pregnancies with a record of receiving tocolytics with no record of PPROM were also included in the preterm labor group. Pregnancies classified as "provider initiated" PTB were those without PPROM or premature labor for whom there was "medical induction", "assisted rupture of membranes", or for whom there was a cesarean delivery at $<37$ weeks of gestation and none of the aforementioned indicators of spontaneous PTB. Rates of PTB (overall and by subtypes and by preeclampsia) were examined by AUC derived probability scores (by deciles) to assess true- and false-positive performance at set cut-points in the training and testing subgroups.

All analyses were done using Statistical Analysis Software (SAS) version 9.3 (Cary, NC). Methods and protocols for the study were approved by the Committee for the Protection of Human Subjects within the Health and Human Services Agency of the State of California, the Institutional Review Board of Stanford University and the Institutional Review Board of the University of California San Francisco.

\section{Results}

Most case and control women in the study identified themselves as Hispanic or White (e.g., 55.8\% of women 


\begin{tabular}{|c|c|}
\hline Interleukins & $\begin{array}{l}\text { Interleukin (IL) - } 1 \text { alpha (IL1A), IL-1 receptor (R) alpha (IL-1RA), IL-1R2, } \\
\text { IL-1 beta (IL-1B), IL-2, IL2RA, IL-4, IL-4R, IL-5, IL-6, IL-6R, glycoprotein } \\
130 \text { (GP130), IL-7, IL-10, IL12p40, IL12p70, IL-13, IL-15, IL-17, IL17F }\end{array}$ \\
\hline Interferons & Interferon alpha (IFNA), IFN beta (IFNB), IFN gamma (IFNG) \\
\hline $\begin{array}{l}\text { Chemokine } \\
\text { Ligands }\end{array}$ & $\begin{array}{l}\text { Chemokine ligand (CCL) - } 2 \text { (CCL2, also known as (AKA): AKA: } \\
\text { monocyte chemotactic protein-1 (MCP-1)); CCL3 (AKA: macrophage } \\
\text { inflammatory protein } 1 \text { alpha (MIP1A)), CCL4 (AKA: MIP-1 beta } \\
\text { (MIP1B)), CCL5 (AKA: regulated on activation, normal T cell expressed } \\
\text { and secreted (RANTES)), CCL7 (AKA: monocyte chemotactic protein-3 } \\
\text { (MCP-3)), CCL11 (AKA: Eotaxin), CXC motif ligand - } 1 \text { (CXCL1) (AKA: } \\
\text { growth-regulated protein alpha (GRO-A)), CXCL5 (AKA: epithelial } \\
\text { neutrophil-activating protein } 78 \text { (ENA-78)), CXCL8 (AKA: IL-8), CXCL9 } \\
\text { (AKA: monokine induced by gamma-interferon (MIG)), CXCL10 (AKA: } \\
\text { interferon-inducible protein-10 (IP-10)) }\end{array}$ \\
\hline $\begin{array}{l}\text { Tumor Necrosis } \\
\text { Factor Alpha } \\
\text { Super Family }\end{array}$ & $\begin{array}{l}\text { Tumor necrosis factor-alpha (TNFA), TNFR1, TNFR2, CD30, CD40-ligand } \\
\text { (CD4OL), sFas Ligand (sFASL), TNF-beta (TNFB), TNF-related apoptosis- } \\
\text { inducing ligand (TRAIL) }\end{array}$ \\
\hline Growth Factors & $\begin{array}{l}\text { Transforming growth factor alpha (TGFA), TGF-beta (TGFB), stem cell } \\
\text { factor (SCF), leukemia inhibitory factor (LIF), platelet-derived growth } \\
\text { factor subunit BB (PDGF-BB), Basic fibroblast growth factor (FGF-basic), } \\
\text { nerve growth factor (NGF), vascular endothelial growth factor (VEGF), } \\
\text { VEGFR1, VEGFR2, VEGFR3, hepatocyte growth factor (HGF) }\end{array}$ \\
\hline $\begin{array}{l}\text { Colony } \\
\text { Stimulating } \\
\text { Factors }\end{array}$ & $\begin{array}{l}\text { Granulocyte-colony stimulating factor (G-CSF), granulocyte } \\
\text { macrophage colony-stimulating factor (GM-CSF), hepatocyte growth } \\
\text { factor, macrophage colony-stimulating factor (M-CSF) }\end{array}$ \\
\hline $\begin{array}{l}\text { Soluble } \\
\text { Adhesion } \\
\text { Molecules }\end{array}$ & $\begin{array}{l}\text { Soluble intercellular adhesion molecule -1 (s1CAM-1), vascular cell } \\
\text { adhesion molecule-1 (sVCAM-1) }\end{array}$ \\
\hline Others & $\begin{array}{l}\text { Leptin, plasminogen activator inhibitor-1 (PAI-1), resistin, receptor for } \\
\text { advanced glycosylation end products (RAGE) }\end{array}$ \\
\hline
\end{tabular}

Fig. 1 Serum markers measured in banked 15-20-week serum samples

with a PTB delivery and $42.5 \%$ of women with a term delivery in the training sample were Hispanic and $47.5 \%$ of women with a PTB delivery and $42.5 \%$ of women with a term delivery in the testing sample were Hispanic). Most women in both the training and testing samples were between 18 and 34 years of age $(67.5-75.0 \%$ across groupings). The majority women with a preterm delivery had a spontaneous PTB $(82.5 \%$ in the training sample and
$75.0 \%$ in the testing sample). The rate of preterm preeclampsia was $15.8 \%$ in the training sample and $22.5 \%$ in the testing sample (Table 1). Crude logistic analyses in the training sample revealed that women with $\mathrm{PTB} \pm$ preeclampsia were significantly more likely $(p<.05)$ than term controls to be low-income (as indicated by MediCal status) (OR 2.07, 95\% CI 1.23-3.48) and to have lower MIP1B levels (OR 0.59, 95\% CI 0.38-0.93) (Supplemental Table 1). 
Table 1 Sample characteristics

\begin{tabular}{|c|c|c|c|c|}
\hline & \multicolumn{2}{|l|}{ Training } & \multicolumn{2}{|l|}{ Testing } \\
\hline & $\begin{array}{l}\text { PTB } \\
n(\%)\end{array}$ & $\begin{array}{l}\text { Term } \\
n(\%)\end{array}$ & $\begin{array}{l}\text { PTB } \\
n(\%)\end{array}$ & $\begin{array}{l}\text { Term } \\
n(\%)\end{array}$ \\
\hline Sample & $\begin{array}{l}120 \\
(100.0)\end{array}$ & $\begin{array}{l}120 \\
(100.0)\end{array}$ & $\begin{array}{l}80 \\
(100.0)\end{array}$ & $\begin{array}{l}80 \\
(100.0)\end{array}$ \\
\hline \multicolumn{5}{|l|}{ Race/ethnicity } \\
\hline Hispanic & $67(55.8)$ & $51(42.5)$ & $38(47.5)$ & $34(42.5)$ \\
\hline White & $39(32.5)$ & $49(40.8)$ & $26(32.5)$ & $35(43.8)$ \\
\hline Asian & $8(6.7)$ & $9(7.5)$ & $11(13.8)$ & $5(6.3)$ \\
\hline Black & $3(2.5)$ & $3(2.5)$ & $3(3.8)$ & $1(1.3)$ \\
\hline Other & 0 & $1(0.8)$ & $2(2.5)$ & 0 \\
\hline \multicolumn{5}{|l|}{ Age (Years) } \\
\hline$<18$ & $1(0.8)$ & $2(1.7)$ & $1(1.3)$ & 0 \\
\hline $18-34$ & $81(67.5)$ & $90(75.0)$ & $56(70.0)$ & $59(73.8)$ \\
\hline$\geq 35$ & $38(31.7)$ & $28(23.3)$ & $23(28.8)$ & $21(26.3)$ \\
\hline \multicolumn{5}{|l|}{ Other (all yes vs. no) } \\
\hline$<12$ years education & $22(18.3)$ & $21(17.5)$ & $16(20.0)$ & $11(13.8)$ \\
\hline $\begin{array}{l}\text { Born in the United } \\
\text { States }\end{array}$ & $76(63.3)$ & $85(70.8)$ & $50(62.5)$ & $54(67.5)$ \\
\hline Low-Income $^{\mathrm{a}}$ & $61(50.8)$ & $40(33.3)$ & $35(43.8)$ & $30(37.5)$ \\
\hline Nulliparous & $54(45.0)$ & $64(53.3)$ & $40(50.0)$ & $39(48.8)$ \\
\hline Reported smoking & $3(2.5)$ & $2(1.7)$ & $1(1.3)$ & $1(1.3)$ \\
\hline Obese & $29(24.2)$ & $21(17.5)$ & $18(22.5)$ & $10(12.5)$ \\
\hline Preexisting diabetes & $3(2.5)$ & $1(0.8)$ & $4(5.0)$ & $1(1.3)$ \\
\hline $\begin{array}{l}\text { Preexisting } \\
\text { hypertension }\end{array}$ & $7(5.8)$ & $3(2.5)$ & $10(12.5)$ & 0 \\
\hline Anemia & $8(6.7)$ & $12(10.0)$ & $11(13.8)$ & $2(2.5)$ \\
\hline IPI $<12$ Months & $24(20.0)$ & $28(23.3)$ & $13(16.3)$ & $14(17.5)$ \\
\hline \multicolumn{5}{|l|}{ Preterm birth subgroups } \\
\hline Spontaneous & $99(82.5)$ & & $60(75.0)$ & \\
\hline Provider initiated & $17(14.2)$ & & $18(22.5)$ & \\
\hline Subtype unknown & $4(3.3)$ & & $2(2.5)$ & \\
\hline$<32$ Weeks & $60(50.0)$ & & $40(50.0)$ & \\
\hline Spontaneous & $53(44.2)$ & & $32(40.0)$ & \\
\hline Provider initiated & $5(4.2)$ & & $8(10.0)$ & \\
\hline Subtype unknown & $2(1.7)$ & & $2(2.5)$ & \\
\hline 32-36 Weeks & $60(50.0)$ & & $40(50.0)$ & \\
\hline Spontaneous & $46(38.3)$ & & $28(35.0)$ & \\
\hline Provider initiated & $12(10.0)$ & & $10(12.5)$ & \\
\hline Subtype unknown & $2(1.7)$ & & $2(2.5)$ & \\
\hline Preeclampsia (any) & $19(15.8)$ & $2(1.7)$ & $18(22.5)$ & $1(1.3)$ \\
\hline$<32$ Weeks & $9(7.5)$ & & $13(16.3)$ & \\
\hline 32-36 Weeks & $10(8.3)$ & & $5(6.3)$ & \\
\hline
\end{tabular}

IPI interpregnancy interval

${ }^{a}$ Receiving assistance for medical services through the California MediCal program (requires an income of $<138 \%$ of federal poverty level)

The final 15 to 20 week $\mathrm{PTB} \pm$ preeclampsia model included maternal age greater than 34-years and lowincome status along with 25 serum biomarkers
(Supplemental Table 2). Serum markers included eight interleukins (IL-1 receptor 2 (IL-1R2), IL-4, IL-4R, IL-5, IL-13, IL-17, IL-17F, and glycoprotein 130 (GP130)), one interferon (interferon (IFN) beta (IFNB)), one factor from the TNFA super family (sFAS ligand (sFASL)), five chemokine ligands (epithelial neutrophil-activating protein 78 (ENA-78), eotaxin, monokine induced by gamma-interferon (MIG), macrophage inflammatory protein 1 beta (MIP1B), and regulated on activation, normal T-cell expressed and secreted (RANTES)), five growth factors (stem cell factor (SCF), platelet-derived growth factor subunit BB (PDGF$\mathrm{BB}$ ), basic fibroblast growth factor (FGF-basic), nerve growth factor (NGF), and vascular endothelial growth factor R3 (VEGFR3)), two colony-stimulating factors (granulocyte-colony-stimulating factor (G-CSF), and macrophage colony-stimulating factor (M-CSF)), as well as PAI1, resistin, and RAGE. Although we found that many of the markers in the final model were highly correlated (VIFs $\geq 2.5$ for 21 of the 24 markers in the final model (IL-1R2, IL4, IL-5, IL-13, IL-17, IL-17F, GP130, IFNB, sFASL, ENA78, eotaxin, MIG, MIP1B, SCF, PDGF-BB, FGF-basic, NGF, VEGFR3, G-CSF, M-CSF, and PAI1) (Supplemental Fig. 3), all of these markers contributed $1 \%$ or more to the c-statistic when included in the model and were, therefore, retained.

When considered in combination using the linear discriminate for $\mathrm{PTB} \pm$ preeclampsia, the 25 target immune and growth factors along with maternal age $>34$ years and low-income status were able to identify more than $80 \%$ of women going on to deliver preterm in the training set (AUC $0.803,95 \%$ CI $0.748-0.858$ ) and $75.0 \%$ of women going on to deliver preterm in the testing set (AUC 0.750, 95\% CI 0.676-0.825) (Table 2, Supplemental Fig. 2). Performance based on the use of combined maternal characteristics and serum markers exceed that based on the use of only characteristics or serum markers (AUC for all preterm birth using maternal age $>34$ and low-income status $=0.620$, 95\% CI (0.553-0.687) in the training set and $\mathrm{AUC}=0.539$ (95\% CI 0.455-0.624) in the testing set; AUC for immune and growth markers only $=0.777(0.719-0.835)$ in the training set and AUC $=0.743(0.667-0.818)$ in the testing set. While performance varied some across PTB subgroups in the training and testing subsets, most AUCs were at or above $80 \%$. One exception was in the training sample where the AUC for PTB 32-36 weeks was 0.790 (95\% CI 0.718-0.862). The largest AUC observed was for preterm preeclampsia $<32$ weeks in the training sample (AUC $=$ $0.953,95 \%$ CI $0.728-0.881$ with an AUC of 0.879 (95\% CI $0.782-0.976$ in the testing sample) (Table 2).

LDA-derived probabilities from the PTB \pm preeclampsia model yielded findings showing that the relationship between risk scores and PTB \pm preeclampsia overall and by subtype was consistent across the training and testing 
Table 2 Performance of mid-pregnancy immune and growth factor preterm birth \pm preeclampsia test (overall and by preterm and preeclampsia subgroups)

\begin{tabular}{cllll}
\hline & \multicolumn{2}{l}{ Training $(n=240)$} & \multicolumn{2}{l}{ Testing $(n=160)$} \\
\hline & AUC & $95 \%$ CI & AUC & $95 \%$ CI \\
All PTB & 0.803 & $0.748-0.858$ & 0.750 & $0.676-0.825$ \\
Spontaneous & 0.806 & $0.748-0.864$ & 0.837 & $0.770-0.903$ \\
Provider initiated & 0.919 & $0.862-0.976$ & 0.858 & $0.771-0.944$ \\
$<32$ & 0.837 & $0.777-0.897$ & 0.806 & $0.717-0.896$ \\
Spontaneous & 0.840 & $0.775-0.904$ & 0.868 & $0.789-0.948$ \\
Provider initiated & 0.927 & $0.818-1.000$ & 0.878 & $0.738-1.000$ \\
34-36 & 0.790 & $0.718-0.862$ & 0.827 & $0.748-0.906$ \\
Spontaneous & 0.801 & $0.723-0.890$ & 0.907 & $0.843-0.971$ \\
Provider initiated & 0.932 & $0.871-0.995$ & 0.893 & $0.796-0.989$ \\
Preeclampsia <37 weeks & 0.889 & $0.822-0.959$ & 0.883 & $0.804-0.963$ \\
$<32$ Weeks & 0.953 & $0.728-0.881$ & 0.879 & $0.782-0.976$ \\
32-36 Weeks & 0.938 & $0.877-0.998$ & 0.950 & $0.882-1.000$ \\
\hline
\end{tabular}

$S P T B$ spontaneous preterm birth, PPROM preterm premature rupture of membranes, $A U C$ area under the receiver operating characteristic curve

subsets with improvements in detection at each lowering of the probability cut point also associated with an increase in term false positives (Fig. 2, Supplemental Table 3). Detection was generally better for PTBs $<32$ weeks and for preterm preeclampsia at each cut point than it was for PTBs from 32 to 36 weeks. For example, $30.8 \%$ of women with PTBs in the training sample and $27.5 \%$ of women with PTBs in the testing sample had probability scores $\geq 0.8$ vs. $3.3 \%$ of women with term birth in the training sample and $1.3 \%$ of term birth in the testing sample (Fig. 2, Supplemental Table 3). Detection at this same cut point was best in women with a PTB $<32$ weeks and in women with preterm preeclampsia in both samples $33.3 \%$ in the training and $27.5 \%$ in the testing samples for PTB $<32$ weeks and $36.8 \%$ in the training sample and $38.9 \%$ in testing sample for preterm preeclampsia) (Fig. 2, Supplemental Table 3).

\section{Discussion}

Results from this study show that when considered in combination, maternal characteristics and serum immune and growth-related markers can be used at 15-20 weeks of gestation to identify women at increased risk for PTB occurring \pm preeclampsia. The resulting LDA PTB \pm preeclampsia model was able to consistently identify more than three and four women going on to deliver preterm across training and testing subsets with the best performance for preterm preeclampsia where AUCs were consistently at or above $88 \%$. LDA-derived probabilities were able to reliably specify a woman's magnitude of risk for $\mathrm{PTB} \pm$ preeclampsia with higher probabilities associated with lower term false-positive rates. For example, while $>60 \%$ of women going on to have a PTB \pm preeclampsia had a 15-20 week LDA-derived probability score $\geq 0.5$ so did $>28 \%$ of pregnancies going on to have a term delivery. While the detection rate was far lower at higher probability cut-points, so was the rate of false positives in term pregnancies. For instance at a LDA-derived probability score $\geq 0.8$, detection rates for PTB were consistently above $25 \%$ and detection rates for PTB with preeclampsia were consistently above $35 \%$ with false-positive rates in pregnancies going to term that were consistently below $5 \%$.

This is the first study that we are aware of that aimed to predict PTB across subtypes \pm preeclampsia. Given that the AUCs from the present study equaled or exceeded those of investigations focused on, for example, spontaneous PTB [14-16] or preeclampsia [22] it appears that such an approach may offer similar predictive capacity and broader applicability over other serum testing approaches. For example, using circulating proteins, Saade and colleagues were able to identify women with a spontaneous PTB $<37$ weeks with an observed AUC of 0.75 [15] and Weiner and colleagues were able to identify women with a spontaneous PTB $<37$ weeks with an observed AUC of 0.76 using cell-free plasma RNAs [17] (compared with an AUC of 0.81 (rounded) for spontaneous PTB in the training set and 0.84 (rounded) in the testing set in the present study). Our results with respect to prediction of preterm preeclampsia also appear to meet or exceed other serum tests for preterm preeclampsia. For example, O'Gorman and colleagues reported an AUC of 0.95 for preeclampsia before 32 weeks and an AUC of 0.87 for any preeclampsia before 37 weeks using 11 to 13 week placental growth factor (PLGF) and pregnancy-associated plasma protein A (PAPP-A) [22]. We observed an AUC for preterm preeclampsia of 0.95 (rounded) in the training set and 0.88 (rounded) in the testing set for preeclampsia $<32$ weeks and we observed an AUC for all preterm preeclampsia ( $<37$ weeks) of 0.89 in the training sample and 0.88 in the testing sample.

While the present test appears to perform as well or better for all births $<37$ weeks than other serum tests that are specific to spontaneous PTB and preeclampsia, two caveats to this should be noted. First, there is some evidence that -omics-based tests for spontaneous PTBs perform better at lower gestational ages than they do at higher gestational ages. Although based on smaller sample sizes for earlier PTBs than in the present study, Saade and colleagues [15] reported an AUC of 0.98 for $<35$ vs. $\geq 35$ weeks and Catonwine and colleagues [16] reported an AUC of 0.89 for 
Fig. 2 True and false-positive rates by probability cut-points based on mid-pregnancy immune and growth factor preterm birth \pm preeclampsia test

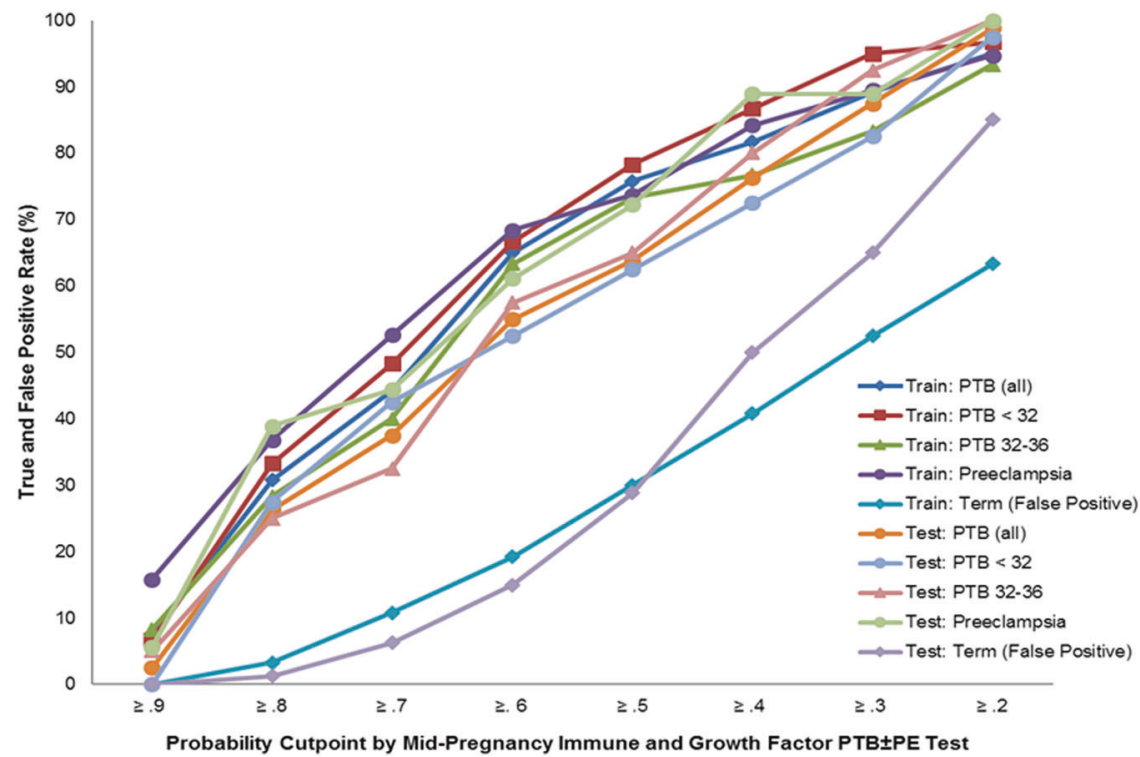

birth at $\leq 34$ weeks. Also, prediction of preterm preeclampsia has been shown to be greatly improved if serum testing is combined with ultrasound measures of mean arterial pressure and uterine artery pulsatility index (AUCs 0.99 for preeclampsia $<32$ weeks and 0.92 for any preeclampsia $<37$ weeks) [22]. Although we contend that the currently presented algorithm represents an improvement over these other methods given that it focus on the commonalities across PTB subtypes and relies on widely available multiplex technology that allows multiple markers to be measured in a single test, it is critical to note that there are likely some benefits to focusing within subtypes. It may be that the present test could be improved further by the inclusion of, for example, a second-tier -omics-based test that addresses other protein-based or metabolic factors. A second-tier test that included ultrasound measures might also increase detection rates for preterm preeclampsia. Such an approach might allow for broad testing for baseline all $\mathrm{PTB} \pm$ preeclampsia risk and second-tier testing that is specifically aimed at early PTBs and preterm preeclampsia with a focus on term false-positive reduction.

The present study focused on the capacity for prediction of PTB \pm preeclampsia and as such, interpretation of causal underpinnings suggested by biomarker patterns should be approached with some caution. Still, given that the serum markers in the final model have established links with poor pregnancy outcomes and close ties to immune function and growth $[23,24,27,28,33-36,38-42]$ some insight into pathophysiological underpinnings is evident. Most notably, the findings from the current study are supportive of the role of perturbation of the cytokine network in the pathogenesis of PTB as proposed by Romero and colleagues [24] - particularly given that the success of the present model in prediction was driven by its reliance on a constellation of markers that were often highly related yet contributed independently to prediction. Study data also realized the assertions of others who have hypothesized that combining cross-pathway markers would increase test performance [43]. By combining cross-way molecular markers with risks like maternal age $>34$ years and low-income status, the model took advantage of well-established maternal risks for PTB [40] along with critical pathway signals.

Our findings of a persistent role in prediction for lowincome status (including when defined by participation in state-sponsored health insurance programs for individuals with incomes near or below the United States poverty line) are consistent with other investigations [44, 45]. We suspect that in our model, poverty is serving as a proxy for unmeasured or underreported factors with links to PTB \pm preeclampsia including, possibly, the presence of nutritional deficits, psycho-social or systemic stress, and greater exposure to potentially harmful substances like tobacco, alcohol, and pollution [44-46]. While we had information about tobacco and alcohol use (as well as drug use) in the study dataset, it is possible that these factors were underreported and as such, that the poverty flag is serving as a proxy for these factors as well as others that may be more common in lower income populations. These patterns support the need for additional research and clinical investigation with these women-particularly with respect to early and potentially modifiable risks. Whatever the case, It is important to note that in the present study these factors alone were poor predictors of preterm birth (with AUCs below $62 \%$ in the training and testing sets) and also that they contributed a relatively small amount of information over and above biomarkers alone (increasing the AUC for 
Table 3 Final 15-20 week linear discriminate for preterm birth (PTB) \pm preeclampsia $^{\mathrm{a}}$

\begin{tabular}{|c|c|c|}
\hline & No preterm birth/PE & Preterm birth/PE \\
\hline Constant & -2229 & -2207 \\
\hline PAI1 & 413.49597 & 411.87715 \\
\hline Resistin & 0.75258 & 1.88708 \\
\hline GP130 & 119.61108 & 118.44810 \\
\hline ENA-78 & -29.26997 & -28.53583 \\
\hline sFASL & 5.54682 & 4.15190 \\
\hline FGF-basic & 200.03457 & 204.35713 \\
\hline G-CSF & 10.37429 & 10.68791 \\
\hline IL-1R2 & -2.50083 & -2.23721 \\
\hline IL-4 & -97.38072 & -94.75076 \\
\hline IL-4R & 23.32864 & 22.69110 \\
\hline IL-5 & 65.86996 & 63.28213 \\
\hline IL-13 & -35.04245 & -33.45918 \\
\hline IL-17 & -114.44812 & -113.34045 \\
\hline IL-17F & -1.80384 & -2.20769 \\
\hline IFNB & 4.26576 & 3.87186 \\
\hline M-CSF & -46.88392 & -47.52238 \\
\hline NGF & 8.44649 & 6.96815 \\
\hline PDGFBB & -23.52635 & -22.59093 \\
\hline RAGE & -4.15909 & -3.75774 \\
\hline $\mathrm{SCF}$ & 40.47520 & 37.72616 \\
\hline VEGFR3 & 14.01668 & 13.74962 \\
\hline Eotaxin & -51.73581 & -53.79304 \\
\hline MIG & 5.47441 & 5.91727 \\
\hline MIP1B & 16.13980 & 14.87844 \\
\hline RANTES & 5.15387 & 4.74134 \\
\hline Age $>34$ years & -15.30541 & -14.42951 \\
\hline Low-income ${ }^{b}$ & 3.66412 & 4.71827 \\
\hline
\end{tabular}

${ }^{a}$ Results presented to the fifth decimal point to allow for complete transparency and replication of complete algorithm

${ }^{b}$ Receiving assistance for medical services through the California MediCal program (requires an income of $<138 \%$ of the federal poverty level)

biomarkers only by $0.026+/-0.058$ in the training set and by $0.008+/-0.075$ in the testing set). As such, it is clear that these factors alone were not the sole drivers of overall risk and may point to more upstream drivers. Nevertheless, it is important to investigate these patterns more completely given potential for modification. These data also suggest that the efficacy of this test would not be diminished in settings characterized by mostly high- or low-income individuals given that molecular factors appear to be the primary drivers of prediction.

While the present study represents an improvement over other tests for PTB \pm preeclampsia-particularly given applicability across PTB subgroups and to larger populations given the use of a random sampling design and the leveraging of multiplex technology available globally, there are important limitations of our work. Most notably, the present study relied on a fairly small number of cases and controls $(n=400)$. While the case number used in the present study is larger than those used to create other tests [21-23] it is still far too small to make firm conclusions about broad testing efficacy and performance. Also, because the current study relied on a population-based sampling design that reflected the true distribution of women participating in prenatal screening by race/ethnicity grouping, we were not powered to look at performance by all race/ ethnicity groupings. Further replication and clinical validation are critical next steps especially in women of Black race/ethnicity and in women living in low-to-middle income countries given their higher risk for PTB $[1,9,10,44]$. It is also important to note that because this study relied on women who had already elected to participate in prenatal screening for aneuploidies and neural tube defects, this sample is biased towards women who made that choice and as such, it is unclear is performance would be the same in all pregnant women.

Given that the model performed well with samples collected at as early as 15-weeks of gestation, we feel confident that the model could be applied at earlier gestational ages. Demonstrating such capacity will be critical given, for example, that the efficacy of aspirin administration to prevent preeclampsia has been shown to work the best if started at $\leq 16$-weeks of gestation [19]. It will also be important to examine how this test behaves in identifying pregnancies that deliver early term (37 and 38 weeks). Given mounting data demonstrating that early term babies are at increased risk for both short- and long-term morbidity [47] and that these women are more likely to deliver preterm in the next pregnancy [48] it would be advantageous to be able to identify these women early in pregnancy in an effort to extend gestation.

It is also important to address some statistical and model complexity issues raised by the current analyses. Specifically, it is notable that the present model is more complex than those that rely on fewer factors. While we do not believe this issue is of considerable consequence given that all markers can be tested simultaneously using multiplex technology (and as such, this complexity has minimal impact on how much serum would be needed for the test or how much it would cost), it does raise some issues with respect to model transparency and comprehension. We have presented the full LDA function used for classification to address this issue (Table 3), however, the fact does remain that the algorithm we developed would have to be applied to any woman's biomarker testing results before they could be used clinically_likely via some electronic platform. It is also of note that some markers in the final model—namely FGF-basic and IL-4 exhibited a particularly large influence 
on the $\mathrm{PTB} \pm$ preeclampsia algorithm while also having large observed confidence intervals in initial multivariate logistic models (Supplemental Table 2). Both of these factors were normally distributed after log transformation and as such, the large risks and confidence intervals observed appeared to be driven by the separation of values for these markers in cases vs. controls after adjustment for the other factors in the model. Given this and the contribution of both to AUC performance these factors were kept in the model, however, these patterns are of note and should be kept in mind as replication and clinical validation efforts move forward. It is possible that in other samples such separation may not be evident and as such, some cost to the performance of the model could occur. No such cost appeared to transpire in the testing set but nonetheless, behavior of these markers should be tracked as efforts move forward. In addition, it should be recognized that because many of the markers in the model are highly correlated but were retained due to their individual contribution to the c-statistic, future larger studies may find that not all markers are required for maximum test performance. To this point, it should also be recognized that any interpretation of underlying etiology based on individual marker-specific findings should be avoided given that the model presented is focused on prediction and is based on a markers and maternal factors considered in combination.

Along with maternal age and poverty status, midpregnancy immune and growth factors measured by a single test reliably identified women who went on to have a $\mathrm{PTB} \pm$ preeclampsia. Such information has the potential to be used to identify women who may benefit from existing and emerging interventions aimed at reducing rates of PTB and preeclampsia [49].

Acknowledgements Data from the California Prenatal Screening Program was obtained through the California Biobank Program (Screening Information System request no. 476). Data were obtained with an agreement that the California Department of Public Health is not responsible for the results or conclusions drawn by the authors of this publication.

Funding Supported by the Bill and Melinda Gates Foundation (OPP52256), NIH/NHLBI (RC2 HL101748, RO1 HD-57192, and R01 HD-52953), the March of Dimes Prematurity Center at Stanford University School of Medicine, the Stanford Child Health Research Institute at Stanford University School of Medicine, the Stanford Clinical and Translational Science Award CTSA to Spectrum (UL1 TR001085), the March of Dimes Prematurity Center-Ohio Collaborative, March of Dimes (6-FY11-261 and FY10-180), and the California Preterm Birth Initiative (PTBi-CA) at the University of California San Francisco Benioff Children's Hospital.

\section{Compliance with ethical standards}

Conflict of interest Dr. Murray works part time for the Bill and Melinda Gates Foundation (one of the funder's of this work). All other authors declare that they have no conflict of interest.
Open Access This article is licensed under a Creative Commons Attribution 4.0 International License, which permits use, sharing, adaptation, distribution and reproduction in any medium or format, as long as you give appropriate credit to the original author(s) and the source, provide a link to the Creative Commons license, and indicate if changes were made. The images or other third party material in this article are included in the article's Creative Commons license, unless indicated otherwise in a credit line to the material. If material is not included in the article's Creative Commons license and your intended use is not permitted by statutory regulation or exceeds the permitted use, you will need to obtain permission directly from the copyright holder. To view a copy of this license, visit http://creativecommons. org/licenses/by/4.0/.

\section{References}

1. Blencowe H, Cousens S, Oestergaard MZ, Chou D, Moller AB, Narwal R, et al. National, regional, and worldwide estimates of preterm birth rates in the year 2010 with time trends since 1990 for selected countries: a systematic analysis and implications. Lancet. 2012;379:2162-72.

2. Liu L, Oza S, Hogan D, Chu Y, Perin J, Zhu J, et al. Global, regional, and national causes of under-5 mortality in 2000-15: An updated systematic analysis with implications for the Sustainable Development Goals. Lancet. 2016;388:3027-35.

3. Vohr BR, Wright LL, Poole WK, McDonald SA. Neurodevelopmental outcomes of extremely low birth weight infants 32 weeks' gestation between 1993 and 1998. Pediatrics. 2005; 116:635-43.

4. Swamy GK, Ostbye T, Skjaerven R. Association of preterm birth with long-term survival, reproduction, and next-generation preterm birth. JAMA. 2008;299:1429-36.

5. Crump C, Sundquist K, Sundquist J, Winkleby MA. Gestational age at birth and mortality in young adulthood. JAMA. 2011;306:1233-40.

6. Parkinson JR, Hyde MJ, Gale C, Santhakumaran S, Modi N. Preterm birth and the metabolic syndrome in adult life: a systematic review and meta-analysis. Pediatrics. 2013;131:e1240-e1263.

7. Tranquilli AL. Early and late-onset pre-eclampsia. Pregnancy Hypertens. 2014;4:241.

8. Lisonkova S, Joseph KS. Incidence of preeclampsia: risk factors and outcomes associated with early- versus late-onset disease. Am J Obstet Gynecol. 2013;209:544. e1-544.

9. Hamilton BE, Martin JA, Osterman MJ. Births: Preliminary Data for 2015. Natl Vital Stat Rep. 2016;65:1-15.

10. Martin JA, Hamilton BE, Osterman JK. Births in the United States, 2016. NCHS Data Brief No. 287, September 2017. Department of Health and Human Services, Washington DC;2017.

11. Jarde A, Lutsiv O, Park CK, Beyene J, Dodd JM, Barrett J, et al. Effectiveness of progesterone, cerclage and pessary for preventing preterm birth in singleton pregnancies: a systematic review and network meta-analysis. BJOG. 2017;124:1176-89.

12. Berghella V, Ciardulli A, Rust OA, To M, Otsuki K, Althuisius S, et al. Cerclage for short cervix on ultrasound in singleton gestations without prior spontaneous preterm birth: a systematic review and meta-analysis of trials using individual patient-level data. Ultrasound Obstet Gynecol. 2017;50:569-77.

13. Petousis S, Margioula-Siarkou C, Kalogiannidis I. Effectiveness of tocolytic agents on prevention of preterm delivery, neonatal morbidity, and mortality: Is there a consensus? a review of the literature. Obstet Gynecol Surv. 2016;71:243-52.

14. Society for Maternal-Fetal Medicine (SMFM) Preterm Birth toolkit. https://www.smfm.org/publications/231-smfm-pretermbirth-toolkit. Accessed on 12 November 2017. 
15. Saade GR, Boggess KA, Sullivan SA, Markenson GR, Iams JD, Coonrod DV, et al. Development and validation of a spontaneous preterm delivery predictor in asymptomatic women. Am J Obstet Gynecol. 2016;214:633. e1-633.e24.

16. Cantonwine DE, Zhang Z, Rosenblatt K, Goudy KS, Doss RC, Ezrin AM, et al. Evaluation of proteomic biomarkers associated with circulating microparticles as an effective means to stratify the risk of spontaneous preterm birth. Am J Obstet Gynecol. 2016;214:631. e1-631.e11.

17. Weiner C, Zhou H, Cuckle H, Ramsey R, Egerman R, Dong Y, et al. Futurebirth ${ }^{\mathrm{TM}}$ - prediction of future preterm birth $33 \mathrm{w}$ and preeclampsia/eclampsia $34 \mathrm{w}$ by $16 \mathrm{w}$ using a novel test in asymptomatic women. AJOG. 2017;216:S196.

18. Voltolini C, Torricelli M, Conti N, Vellucci FL, Severi FM, Petraglia F. Understanding spontaneous preterm birth: from underlying mechanisms to predictive and preventive interventions. Reprod Sci. 2013;20:1274-92.

19. Bujold E, Roberge S, Nicolaides KH. Low-dose aspirin for prevention of adverse outcomes related to abnormal placentation. Prenat Diagn. 2014;34:642-8.

20. Rolnik DL, Wright D, Poon LC, O'Gorman N, Syngelaki A, de Paco Matallana C, et al. Aspirin versus placebo in pregnancies at high risk for preterm preeclampsia. N Engl J Med. 2017;377: 613-22.

21. D'Silva A, Fyfe R, Hyett J. First trimester prediction and prevention of adverse pregnancy outcomes related to poor placentation. Curr Opin Obstet Gynecol. 2017;29:367-74.

22. O'Gorman N, Wright D, Poon LC, Rolnik DL, Syngelaki A, Wright A, et al. Accuracy of competing-risks model in screening for pre-eclampsia by maternal factors and biomarkers at 11-13 weeks' gestation. Ultrasound Obstet Gynecol. 2017;49:751-5.

23. Bhat G, Williams SM, Saade GR, Menon R. Biomarker interactions are better predictors of spontaneous preterm birth. Reprod Sci. 2014;21:340-50.

24. Romero R, Grivel JC, Tarca AL, Chaemsaithong P, Xu Z, Fitzgerald $\mathrm{W}$, et al. Evidence of perturbations of the cytokine network in preterm labor. Am J Obstet Gynecol. 2015;213:836. e1- e11.

25. Nourollahpour Shiadeh M, Behboodi Moghadam Z, Adam I, Saber V, Bagheri M, Rostami A. Human infectious diseases and risk of preeclampsia: an updated review of the literature. Infection. 2017;45:589-600. https://doi.org/10.1007/s15010-017-1031-2. epub ahead of print 2 June 2017.

26. Jafri S, Ormiston ML. Immune regulation of systemic hypertension, pulmonary arterial hypertension and preeclampsia: shared disease mechanisms and translational opportunities. Am J Physiol Regul Integr Comp Physiol. 2017;313:R693-R705. https:// doi.org/10.1152/ajpregu.00259.2017. e-pub ahead of print 4 Oct 2017.

27. Yang J, Pearl M, DeLorenze GN, Romero R, Dong Z, JelliffePawlowski L, et al. Racial-ethnic differences in midtrimester maternal serum levels of angiogenic and antiangiogenic factors. Am J Obstet Gynecol. 2016;215:359. e1-9.

28. Lee SE, Kim SC, Kim KH, Yoon MS, Eo WK, Kim A, et al. Detection of angiogenic factors in midtrimester amniotic fluid and the prediction of preterm birth. Taiwan J Obstet Gynecol. 2016;55:539-44.

29. California Biobank Program (CBP). https://www.cdph.ca.gov/ Programs/CFH/DGDS/Pages/cbp/default.aspx. Accessed 12 November 2017.

30. Jelliffe-Pawlowski LL, Ryckman KK, Bedell B, O'Brodovich HM, Gould JB, Lyell DJ, et al. Elevated mid-pregnancy tumor necrosis factor-alpha (TNF- $\alpha$ ) and lipid patterns suggestive of hyperlipidemia in pregnancies resulting in early preterm birth. Am J Obstet Gynecol. 2014;211:141. e1-9.

31. Jelliffe-Pawlowski LL, Baer RJ, Currier RJ, Lyell DJ, Blumenfeld YJ, El-Sayed YY, et al. Early-onset severe preeclampsia by first trimester pregnancy-associated plasma protein A and total human chorionic gonadotropin. Am J Perinatol. 2015;32:703-12.

32. American Medical Association. International Classification of Diseases; Physician, Ninth Revision Clinical Modification. 9th edn. Chicago: American Medical Association; 2008.

33. Menon R, Bhat G, Saade GR, Spratt H. Multivariate adaptive regression splines analysis to predict biomarkers of spontaneous preterm birth. Acta Obstet Et Gynecol Scand. 2014;93:382-91.

34. Zhang Z, Gao Y, Zhang L, Jia L, Wang P, Zhang L, et al. Alterations of IL-6, IL-6R and gp130 in early and late onset severe preeclampsia. Hypertens Pregnancy. 2013;32:270-80.

35. Dinarello CA. Historical insights into cytokines. Eur J Immunol. 2007;37(Suppl 1):S34-45.

36. Turner MD, Nedjai B, Hurst T, Pennington DJ. Cytokines and chemokines: At the crossroads of cell signalling and inflammatory disease. Biochim Biophys Acta. 2014;1843:2563-82.

37. The Human Immune Monitoring Center (HIMC). http://iti.stanford.edu/himc/immunoassays.html (Luminex). Accessed 12 November 2017.

38. Mor G, Aldo P, Alvero AB. The unique immunological and microbial aspects of pregnancy. Nat Rev Immunol. 2017;17:469-82.

39. Morgan JA, Bombell S, McGuire W. Association of plasminogen activator inhibitor-type 1 (-675 4G/5G) polymorphism with preeclampsia: systematic review. PLoS ONE. 2013;8:e56907.

40. Ryckman KK, Williams SM, Krohn MA, Simhan HN. Interaction between interleukin- 1 receptor 2 and Toll-like receptor 4, and cervical cytokines. J Reprod Immunol. 2011;90:220-6.

41. Jonsson Y, Rubèr M, Matthiesen L, Berg G, Nieminen K, Sharma $\mathrm{S}$, et al. Cytokine mapping of sera from women with preeclampsia and normal pregnancies. J Reprod Immunol. 2006;70:83-91.

42. Cappelletti M, Presicce P, Lawson MJ, Chaturvedi V, Stanklewicz TE, Vanoni S, et al. Type I interferons regulate susceptibility to inflammation-induced preterm birth. JCI Insight. 2017;2:e91288.

43. Chan RL. Biochemical markers of spontaneous preterm birth in asymptomatic women. Biomed Res Int. 2014;2014:64081.

44. Jelliffe-Pawlowski LL, Baer RJ, Blumenfeld YJ, O'Brodovich HM, Gould JB, Druzin ML, et al. Maternal characteristics and mid-pregnancy serum biomarkers as risk factors for subtypes of preterm birth. BJOG. 2015;122:1484-93.

45. Janevic T, Stein CR, Savitz DA, Kaufman JS, Mason SM, Herring AH. Neighborhood deprivation and adverse birth outcomes among diverse ethnic groups. Ann Epidemiol. 2010;20:445-51.

46. Smith LK, Draper ES, Evans TA, Field DJ, Johnson SJ, Manktelow BN, et al. Associations between late and moderately preterm birth and smoking, alcohol, drug use and diet: a populationbased case-cohort study. Arch Dis Child Fetal Neonatal Ed. 2015;100:F486-491.

47. Buckles K, Guldi M. Worth the wait? The effect of early term birth on maternal and infant health. J Policy Anal Manag. 2017;36:748-72.

48. Yang J, Baer RJ, Berghella V, Chambers C, Chung P, Coker T, et al. Recurrence of preterm birth and early term birth. Obstet Gynecol. 2016;128:364-72.

49. Ireland DJ, Nathan EA, Li S, Charles AK, Stinson LF, Kemp MW, et al. Preclinical evaluation of drugs to block inflammationdriven preterm birth. Innate Immun. 2017;23:20-33. 